\title{
Quantitative and qualitative evaluation of the Wide Volume (WV) versus Helical acquisition on a 320-detector row computed tomography for the exploration of the abdominopelvic region in adults
}

\author{
Catherine Roy ${ }^{1 *}$, Raphael Quin ${ }^{1}$, Aïssam Labani ${ }^{1}$, Pierre Leyendecker ${ }^{1}$, Luc Mertz $^{2}$ and Mickael Ohana ${ }^{1}$ \\ ${ }^{1}$ Department of Radiology, University Hospital of Strasbourg - New Civil Hospital, Strasbourg Cedex, France \\ ${ }^{2}$ Medical Physics Department, University Hospital of Strasbourg - New Civil Hospital, Strasbourg Cedex, France
}

\begin{abstract}
Purpose: To evaluate the best protocol to be used in WV mode for the abdominopelvic region in terms of radiation dose and image quality by comparison with the helical mode.

Methods and materials: This study was performed on a 320 x $0.5 \mathrm{~mm}$ detector row CT unit. By using several simulations on topograms data and phantoms analysis, we registered CTDIvol. Then, a clinical study on CTUrography was performed, each patient being its own standard.

Results: The collimation of $10 \mathrm{~cm}(200 \times 0.5 \mathrm{~mm})$ with 5 volumes had the lowest CTDIvol. This can be explained by several physical phenomena. In the clinical study, the average radiation dose reduction was found to be at $44.03 \% \pm 0.36 \%(\mathrm{p}<0.001)$. Concerning the qualitative evaluation of the stitching of different volumes and the overall image quality, it was found excellent.
\end{abstract}

Conclusion: Wide Volume CT scanning using 5 volumes of $10 \mathrm{~cm}(200 x 0.5 \mathrm{~mm})$ significantly reduces the radiation dose with an excellent image quality.

Abbreviations: CTDIvol: CT dose index; WV: Wide Volume mode; BMI: Body Mass Index; ROI: Region of interest; SNR: Signal to noise ratio

\section{Introduction}

Recent advances in the CT technology have made a large 320 detectors row available for clinical use. This new technology with a length of up to $16-\mathrm{cm}$ of coverage in the $\mathrm{z}$-axis with a single non-helical rotation allows the exploration of an entire organ such as the heart or the kidney in one rotation. With the short time of one acquisition there is a decreasing of motion artifacts as well as a significant reduction of the exposure dose [1-3] compared to the reference helical acquisition. This type of acquisition called Wide Volume (WV) has been previously used with a collimation of $16-\mathrm{cm}$ in clinical practice for cardiac $[4,5]$, pediatric [6,7] and lumbar [3] imaging as well as to study the perfusion of organs. However, to the best of our knowledge, this promising technique has not yet been applied with multiple volumes for the exploration of the abdominopelvic region in adults.

The purpose of this study was to determine which is the best compromise of $\mathrm{WV}$ parameters to be used to cover a large anatomical region in regard to the scan time, the radiation dose and the image quality for a clinical application by a direct comparison with the conventional helical mode.

\section{Methods and materials}

This monocentric prospective study has been conducted within the radiology department of our academic hospital, in cooperation with the Medical Physics Department. Our institutional ethical committee reviewed and approved our investigation, and no written patient consent was necessary. Patients were orally informed of our new procedure for which details as well as our goal had been explained.

This study was performed using a $320 \times 0.5 \mathrm{~mm}$ detector row CT unit (Aquilion ONE Vision Edition, Toshiba Medical Systems, Otawara, Japan) and was divided into two parts.

Part 1: Topogram-based collimation simulation and Phantoms analysis

The objective was to determine which is the optimal collimation parameters regarding the radiation dose using the topograms data of patients before scanning. Twenty-five patients (mean age: $52 \pm 9$ years) with a body mass index between 20 and $25 \mathrm{~kg} / \mathrm{m}^{2}$ and a height between $160 \mathrm{~cm}$ and $180 \mathrm{~cm}$ referred to our department for an abdominopelvic exploration were included. The acquisition length extended from diaphragm to the pubic bone. We simulated on each topogram the outcome in terms of radiation dose (CTDIvol) and scanning time (in

Correspondence to: Catherine Roy, Department of Radiology, University Hospital of Strasbourg - New Civil Hospital, Strasbourg Cedex, France; Tel: +333369550731; Fax: +3333691815; E-mail: catherine.roy@chru-strasbourg.fr

Key words: Multidetector CT, Radiation dose, Wide-volume CT, Helical CT

Received: October 22, 2017; Accepted: November 13, 2017; Published: November 16, 2017 
Roy C (2017) Quantitative and qualitative evaluation of the Wide Volume (WV) versus Helical acquisition on a 320-detector row computed tomography for the exploration of the abdominopelvic region in adults

seconds) of 6 different combinations, ranging from $120 \times 0.5 \mathrm{~mm}(6$ $\mathrm{cm})$ to $320 \times 0.5 \mathrm{~mm}(16 \mathrm{~cm})$ with an increment of $20 \mathrm{~mm}$ and the helical acquisition. All other parameters $(120 \mathrm{kV}$, field of view, rotation time: $0.5 \mathrm{~s}$, slice thickness: $1 \mathrm{~mm}$ ) were kept identical.

The CTDIvol parameter was considered for both acquisition modes as there is no pitch in WV mode [8].

A second step was performed in order to objectively determine the factors of the radiation dose reduction in the $\mathrm{WV}$ acquisition. To do this, we quantified the absorbed dose due to the primary beam by mean of a radiosensitive film (Gafchromic ${ }^{\circledR} \mathrm{XR}-\mathrm{QA}$ ) positioned between two $10 \mathrm{~cm}$ thick plates of polymethylmethacrylate (PMMA) to get an homogeneous phantom. This phantom was scanned with two types of acquisitions with the same coverage length: a WV mode with 3 consecutive volumes of $8 \mathrm{~cm}(160 \times 0.5 \mathrm{~mm})$ and a helical mode, keeping all other acquisition parameters identical. For the WV acquisition, the 3 volumes were stitched together using the dedicated software of the CT unit.

The dose profiles for the WV and the helical acquisition were then generated from the optical densities and read on a radiosensitive film.

\section{Part 2: Clinical study}

After recording the results of the first part of this study, a prospective clinical evaluation was performed, including 45 median size patients (mean age: $68 \pm 14$ years, BMI ranged from 20 to 25 $\mathrm{kg} / \mathrm{m}^{2}$; height between 160 and $180 \mathrm{~cm}$ ); who were addressed to our radiological department for a CT Urography in several clinical context: exploration of a microscopic hematuria (13 cases), follow up of a bladder excretory tumour (22 cases) and evaluation of urinary stones (10 cases). As a part of our routine protocol, all patients underwent first an abdominopelvic acquisition without contrast medium using the conventional helical mode and the excretory phase was performed with the WV mode at the collimation of $10 \mathrm{~cm}(200 \times 0.5 \mathrm{~mm})$ and 5 volumes to cover entirely this anatomical zone. So that each patient is its own control. The acquisition extended from the diaphragm to the pubis and the explored length was exactly the same between the two scan modes. This was thoroughly controlled at the CT-console using the initial tomogram for each patient. Parameters are detailed in Table 1.

The active collimation was used in helical mode. After the WV acquisition, an automatic stitching of the volumes is realized by the computer and then the CT technician started an additional manual stitching of the volume using a dedicated software at the CT scanner console.

\section{Imaging analysis}

Quantitative analysis: The CTDIvol with standard deviation were recorded for each acquisition mode.

In another reading session, readers recorded on the workstation (Vitrea, Vital Images, USA), the signal to noise ratio (SNR) with standard deviation by mean of a uniform circular ROI of $10 \mathrm{~mm}$ in diameter $\left(78.5 \mathrm{~mm}^{2}\right)$ located inside the psoas muscle at the level of the fourth vertebra on the helical as well as on the WV images.-

Qualitative analysis: The qualitative image analysis was performed by two readers: a fellow in radiology and a senior radiologist (over 10 years of experience in the field of uroradiology). Readers were asked to independently analyse the overall image quality of WV images using a four points subjective scale $(1:$ poor, 2 : fair, 3 : good, $4:$ excellent) and also to evaluate the quality of the fusion of the volumes in WV mode based on the criteria of continuity of the whole ureter with a three points scale : 1 (no visible discontinuity of the ureter) ; 2 (presence of tiny discontinuity of the ureter that does not interfere with the interpretation of the urinary tract) ; 3 (large discontinuity that does not allow an appropriate evaluation of the urinary tract at this level). Disagreement between readers was resolved by consensus with a third experienced radiologist of over 20 years of experience in this field.

\section{Statistical analysis}

Interobserver agreement between the two readers was determined by a kappa test. A kappa value of 0.20 or less indicated slight agreement; $0.21-0.40$, fair agreement; $0.41-0.60$, moderate agreement; $0.61-0.80$, substantial agreement; and 0.81-0.99, almost perfect agreement.

After ensuring normal distribution by Shapiro- Wilk's test, we used the Mann-Whitney $U$ test to compare the recorded radiation dose and SNR values between the two scan modes.

The statistical analysis was done using the XLSTAT statistical software (Addinsoft, version 2015.4.1 for Windows, NY, USA) package.

\section{Result}

\section{Part 1: Topogram-based collimation simulation}

The 25 included patients had a mean body mass index of $23.9 \pm$ $0.8 \mathrm{~kg} / \mathrm{m}^{2}$.

At constant parameters, the collimation of $10 \mathrm{~cm}(200 \times 0.5$ $\mathrm{mm})$ had the lowest CTDIvol equal to $2.78 \pm 0.35 \mathrm{mGy}(\mathrm{p}<0.01)$. An average increase of CDTIvol by $21 \%$ and $18 \%$ was observed for larger collimations of $16 \mathrm{~cm}(320 \times 0.5 \mathrm{~mm})$ and $14 \mathrm{~cm}(280 \times 0.5 \mathrm{~mm})$, respectively. Similarly, an average increase of CDTIvol by $27 \%$ was noted when a smaller collimation of $6 \mathrm{~cm}(120 \times 0.5 \mathrm{~mm})$ was used (Figure 1).

On the helical mode, the CTDIvol was equal to $4.38 \pm 0.48 \mathrm{mGy}$ at a similar coverage length, corresponding to a significant increase of $57 \%(p<0.01)$.

For the coverage of this anatomical region, the reference acquisition time using the helical acquisition was $7.5 \pm 0.7$ seconds for a mean z-coverage of $438 \pm 12 \mathrm{~mm}$.

The acquisition time with the $\mathrm{WV}$ acquisition to cover this adequate anatomical region was dependent on the collimation (Table 2). These values are explained by a mean interval time of approximately 2 seconds in-between each stack, corresponding to the table movement.

We obtained a different scan range for each WV collimation from 425 to $497 \mathrm{~mm}$ to cover the appropriate region of interest.

The value of the overlapping between the different collimation was varying from $11.5 \mathrm{~mm}$ with a collimation of $6 \mathrm{~cm}$ to $24.5 \mathrm{~mm}$ with a collimation of $16 \mathrm{~cm}$, respectively. For the lowest radiation dose option $(10 \mathrm{~cm})$ the value of the overlapping was $15 \mathrm{~mm}$.

We observed that the automated tube current modulation was less precise with the WV mode than with the helical mode. (Figure 2)

The analysis of the phantom radio-sensitive film dose profiles curves provided insights several physical phenomena of the interaction of the ionizing radiations. (Figure $3 \mathrm{a}, \mathrm{b}$ )

\section{Part 2: Clinical study}

The 45 included patients had a mean body mass index of $22.2 \pm 1.0$ $\mathrm{kg} / \mathrm{m} 2$. There was no abnormality in 37 cases and urinary calculus 
Roy C (2017) Quantitative and qualitative evaluation of the Wide Volume (WV) versus Helical acquisition on a 320-detector row computed tomography for the exploration of the abdominopelvic region in adults

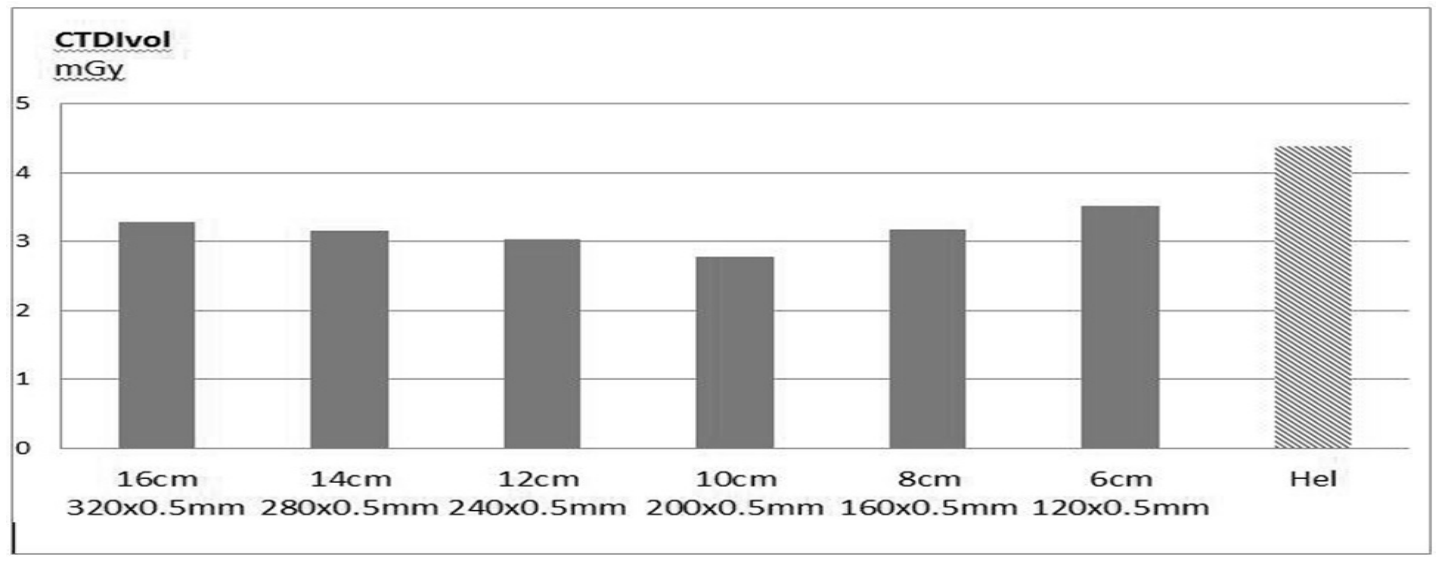

Figure 1. Variations of the radiation dose according different collimation with the WV mode compared to that of the helical mode for the same coverage length.

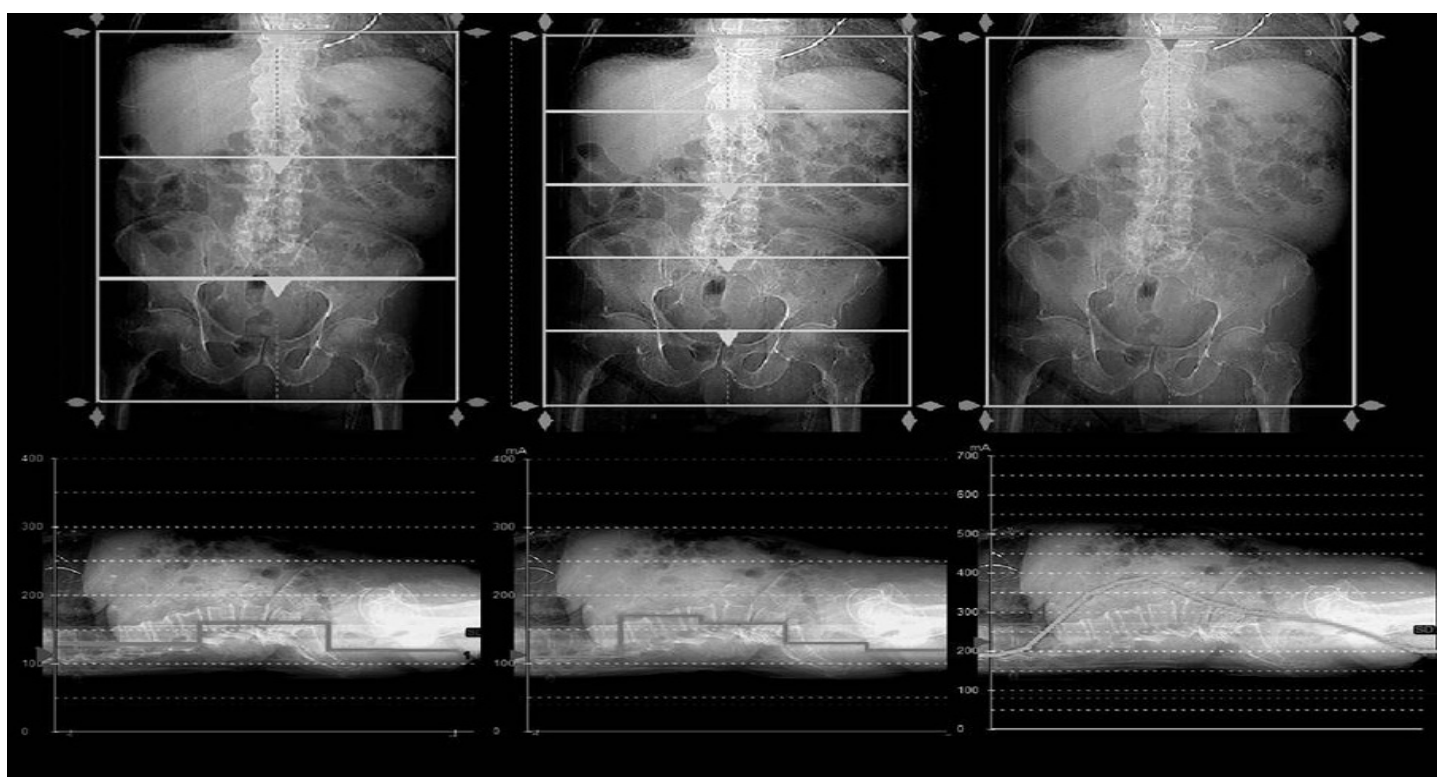

Figure 2. Results of tube current modulation with different collimations in Wide Volume mode (left. 3 volumes, middle: 5 volumes) and with the Helical mode (right).

Table 1. Acquisition parameters for Helical and Wide Volume modes

\begin{tabular}{|c|c|c|}
\hline Acquisition mode & Wide-Volume mode & Helical mode \\
\hline Collimation (mm) & Variablex0.5 & $100 \times 0.5$ \\
\hline Tube voltage (kV) & 120 & 120 \\
\hline FOV (mm) & $320-370$ & $320-370$ \\
\hline Rotation time (s) & 0.5 & 0.5 \\
\hline Slice Thickness (mm) & $1 \mathrm{~mm}$ & $1 \mathrm{~mm}$ \\
\hline Overlapping (mm) & Variable & $0.8 \mathrm{~mm}$ \\
\hline Pitch & No Pitch & 0.8 \\
\hline Itérative Reconstruction & AIDR 3D standard & AIDR 3D standard \\
\hline
\end{tabular}

Table 2. Parameters at different collimations with the corresponding acquisition time in Wide Volume mode.

\begin{tabular}{|c|c|c|c|c|}
\hline $\begin{array}{l}\text { Collimation } \\
\text { (cm) }\end{array}$ & $\begin{array}{c}\text { Number of } \\
\text { stack }\end{array}$ & $\begin{array}{l}\text { Overlapping } \\
(\mathbf{m m})\end{array}$ & $\begin{array}{l}Z \text { coverage } \\
(\mathrm{mm})\end{array}$ & $\begin{array}{c}\text { Acquisition } \\
\text { time (sec) }\end{array}$ \\
\hline $6(120 \times 0.5 \mathrm{~mm})$ & 9 & 11.5 & 448 & 20 \\
\hline $8(160 \times 0.5 \mathrm{~mm})$ & 7 & 14.3 & 474 & 15.5 \\
\hline $10(200 x 0.5 \mathrm{~mm})$ & 5 & 15 & 440 & 10.5 \\
\hline $12(240 \times 0.5 \mathrm{~mm})$ & 4 & 18.3 & 425 & 8 \\
\hline $14(280 \times 0.5 \mathrm{~mm})$ & 4 & 21 & 497 & 8 \\
\hline $16(320 \times 0.5 \mathrm{~mm})$ & 3 & 24.5 & 431 & 5.9 \\
\hline
\end{tabular}

disease was correctly evaluated in 8 cases. The additional time (3s) due the acquisition in WV mode was not considered as significant. The result of the automatic stitching was obtained in one minute. The dedicated software for the manual stitching was very easy to use and the time for computing was less than two minutes.

\section{Quantitative evaluation: (Figure 4)}

The average CTDIvol in WV mode was $2.39 \pm 0.25 \mathrm{mGy}$ and $4.27 \mathrm{mGy} \pm 0.48$ in Helical mode. The average dose reduction was $44.03 \% \pm 0.36 \%(\mathrm{p}<0.001)$ for our cohort of patients by using 5 volumes with a collimation of $10 \mathrm{~cm}(200 \times 0.5 \mathrm{~mm})$ to cover the whole abdominopelvic region.

The acquisition time was added by few seconds, but this does not in clinical practice impact our examination time.

The SNR measured at the psoas muscle was significantly lower ( $\mathrm{p}$ $=0.045)$ on images in VW mode versus helical mode $17.7 \pm 1.9$ vs 20.3 \pm 2.5 , respectively. But this was not significant for the visual routine analysis. 
Roy C (2017) Quantitative and qualitative evaluation of the Wide Volume (WV) versus Helical acquisition on a 320-detector row computed tomography for the exploration of the abdominopelvic region in adults

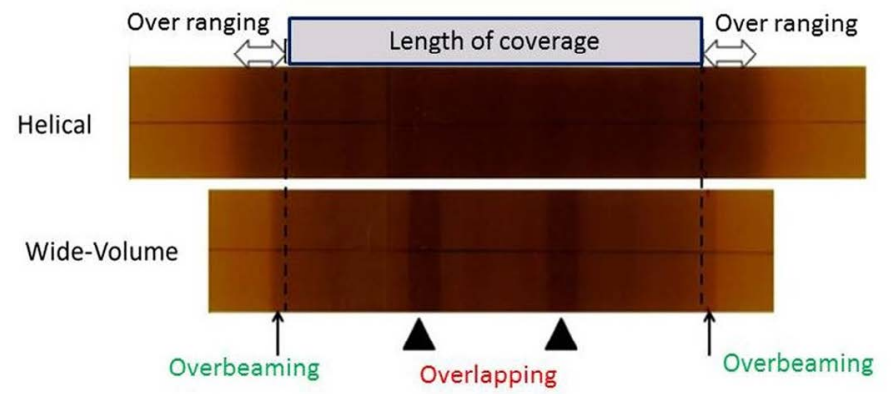

3a. Aspect of the radio-sensitive films. In Wide-Volume mode, the 3 volumes are of the same size. The two central areas appear as 2 vertical brown stripes regularly spaced between volumes (black arrowhead), correspond to the zone of overlapping of the volumes with a localized higher radiation dose. The black arrows represent the over beaming (penumbra) which is relatively limited In Helical mode, the over ranging is visible at both ends of the segment as a larger and poorly limited zone (white arrow).

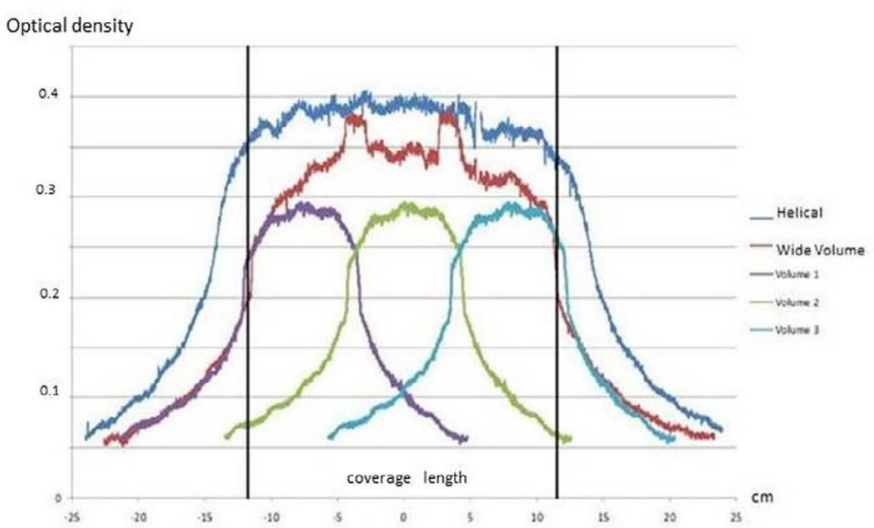

Figure 3b. Dose-profiles extrapolated from radio-sensitive film with an optical scanner: The vertical black lines represent the scan range of $21.5 \mathrm{~cm}$. In the Wide-volume curve (red) the two peaks correspond to the region of overlapping of the two volumes. At the ends of the scan range, the fall in the dose delivered is much more linear and faster in Wide-Volume than in helical mode.

Figure 3. Radio-sensitive film Gafchromic $($ XR-QA and dose-profiles curves. On the radiosensitive films the coverage length was equal to $21.5 \mathrm{~cm}$ for both modes.

\section{Qualitative evaluation: (Figures 5, 6)}

Agreement between the two readers was excellent with a $\kappa$ correlation coefficient as high as 0.89 . The intervention of the third reader was required in 2 cases. For image quality, our results according the subjective scale were as follows: score 1: poor (no case), score 2: fair (no case), score 3: good (14 cases) and score 4: excellent (31cases).

Concerning the quality of the stitching based on the continuity of the ureter, it was found discrepancies between the different volumes. We have found between the first and second volume at the upper level of the abdominopelvic region the following results: $82 \%, 15 \%$ and $3 \%$ of cases for point 1, 2 and 3, respectively. For the three lower volumes, the final stitching was always excellent and rated 1 in 44 cases (except in one case with point 2).) No cases were uninterpretable. The diagnosis was performed without any ambiguity.

\section{Discussion}

Newer developments in CT imaging including advanced technology and easier accessibility has allowed more pertinent clinical indications. Considering this fact, the radiation dose reduction has become an important public health issue, especially for the youngest patients.
Helical scanning is currently the standard acquisition method in clinical practice.

The WV mode has been previously studied for one or two 16 $\mathrm{cm}$ volumetric scanning, to cover a region up to $28 \mathrm{~cm}$. It combines sequential single rotations with table motion in-between. Our study is the first, to the best of our knowledge, to investigate the technical aspect and the clinical use of this mode with more than two acquisitions to cover a broader distance [9].

In agreement with other papers, we have found that a significant reduction of the radiation dose can be obtained with it, but it is highly correlated with the choice of the collimation. For an abdominopelvic exploration, the best compromise is a collimation of $10 \mathrm{~cm}(200 \times 0.5$ $\mathrm{mm}$ ). With this collimation, the acquisition time remains acceptable, even if significantly longer ( +3 seconds) than with the helical mode.

This dose-saving effect has been found in several studies conducted with pediatric phantoms $[2,6]$ and has been attributed to the lack of $z$-axis overranging and to the decreased overbeaming effects compared with those during the helical acquisition.

The radiation dose reduction obtained with the WV mode compared to the helical mode can be explained by several physical phenomena.

The tube current modulation technique in the $\mathrm{Z}$-axis used in routine clinical practice consists of modulating the tube current $(\mathrm{mA})$ of the radiation in the $\mathrm{Z}$-axis, depending on the density and thickness of the tissues to be acquired.

In helical mode, there is a very precise modulation, performed at every rotation, which reduces the dose by $43 \%$ to $66 \%$ when compared to acquisitions without modulation on abdominopelvic scans according to Kalra et al. [10].

The tube current modulation in Z-axis in WV mode is identical within each volume, but varies between 2 volumes acquired and could be problematic for areas with a high-density difference such as the

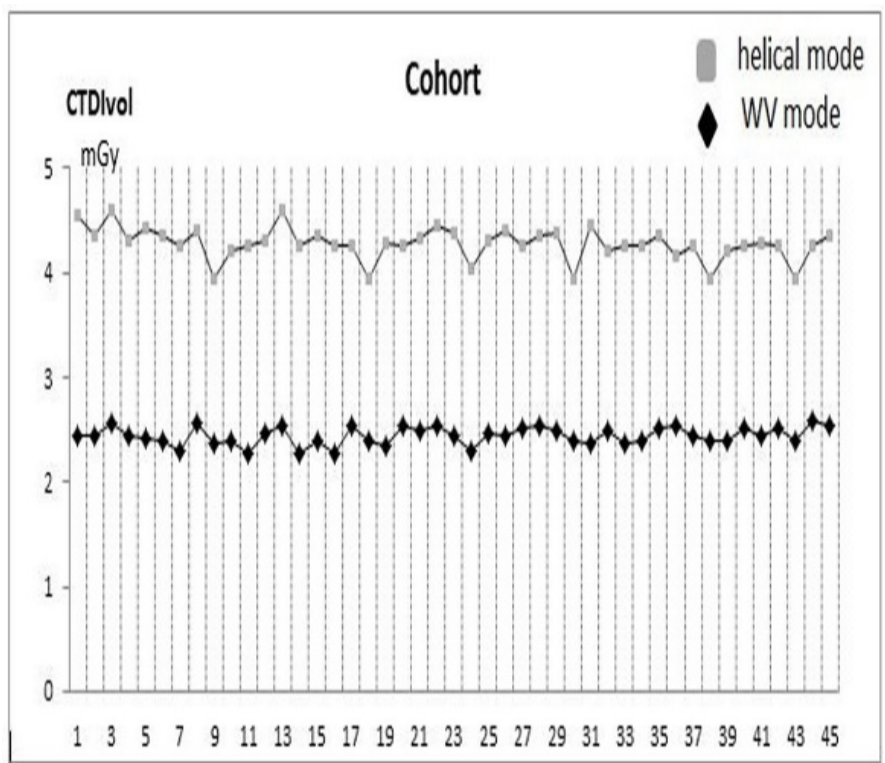

Figure 4. Clinical cohort of 45 patients. The different values of the radiation dose between Helical (unenhanced phase) and WV mode (excretory phase) with a collimation of $10 \mathrm{~cm}$ for each patient. 
Roy C (2017) Quantitative and qualitative evaluation of the Wide Volume (WV) versus Helical acquisition on a 320-detector row computed tomography for the exploration of the abdominopelvic region in adults

shoulders-neck or pelvis [11]. Consequently, a smaller collimation in WV enables a better tailoring of the tube-current modulation, and drives a reduction in the radiation dose. In the study of Gervaise in 2010 [3], there was a difference of contrast between each volume, related to the difference of charge between them. This phenomenon was not observed on our radiosensitive films as well as in our clinical study where there was no inappropriate difference of contrast between the volumes (Figures 5, 6).

The overranging is an extra-rotation required on both sides of the planned scan, necessary to acquire the integrity of the first and last images of the region. Due to the nature of the helical mode, the overranging mechanism is always present and responsible of an overradiation to radiosensitive organs such as the thyroid or testis. With the advent of MDCT, it is playing an increasingly important role in the dose delivered to the patient.

The active collimation is a technique which reduces partially the overranging phenomenon [12]. Despite its use, the phenomenon of overranging is clearly evident on radiation-sensitive film and the dose profile of the helical mode. There is a complete absence of overranging phenomenon in WV mode (Figure 3a).

The phenomenon of penumbra or overbeaming corresponds to a certain quantity of radiation that does not reach the active detectors. As it is necessary to expose all detectors homogeneously, the primary $\mathrm{X}$-ray beam needs to be wider than the detector ring in the $\mathrm{Z}$-axis. The penumbra effect is more important on small primary collimations, and decreases with an increase in the number of detectors. Hence it has become less important with the development of very large detector scanners [13].

The overbeaming is repeated for each rotation of the tube in both helical and WV modes. As long as we use large collimations in WV mode, the phenomenon of overbeaming is rare. As we reduce the length of our volumes, we increase the phenomenon of overbeaming by the repetition of smaller volumes.

To achieve the continuity of images without loss of information a volume overlap is essential. This is responsible for additional irradiation inside this overlapping area (Figure $3 a, 3 b$ ).

In 2014, Liao et al. [14] showed a proportional increase of the overlapping phenomena to the number of acquired volumes. At a certain threshold $(<8 \mathrm{~cm})$, repeated overlapping of volumes increases the delivered dose, and small collimations promote overbeaming. These two phenomena are responsible for a relative over radiation.

Our study showed that to cover a large anatomical zone, the best compromise between all these parameters is to use a collimation of 10 $\mathrm{cm}$, because it is the result of several factors: the automated tube-current modulation is precise enough, compared to greater collimations like 12 or $16 \mathrm{~cm}$; the overranging is absent compared to the helical mode; the overbeaming is relatively low, compared to lower collimations like 6 and $8 \mathrm{~cm}$ and the overlapping is relatively modest, compared to the lower collimations.

The final acquisition time is proportional with the number of volumes. In our evaluation, four volumes give the same acquisition time as the helical mode; but the radiation dose was slightly higher.

However, this WV mode does not allow to adapt precisely the coverage length because this method is to accumulate several regular lengths. But, in our evaluation on topograms it was always easy to get the useful area for a clinical application, especially for an abdominal purpose.

Our study demonstrated that in accordance with the prior literature [15], the WV mode was associated with a lower signal to noise ratio. However, as it was not significant on our subjective clinical study, we believe that the increased noise observed in the volume scanning has been partially corrected by the algorithms of our CT scanner.

Even if the radiation dose recorded in our helical mode is in the lowest range of current thresholds for abdominopelvic evaluation due to the association of a recent iterative reconstruction algorithm, the tube current modulation technique in the three axis and the use of the active collimation, the $\mathrm{WV}$ acquisition with our best collimation of 10 $\mathrm{cm}$ provides a gain in dose reduction of almost fifty per cent. On the other hand, it should be possible to improve the performance of this $\mathrm{WV}$ acquisition in terms of radiation dose by using a shorter rotation time of $0.35 \mathrm{~s}$ or $0.275 \mathrm{~s}$ and a lower voltage of $100 \mathrm{kVp}$.

The lower quality of the fusion between the two first volumes can be attributed to tiny breathing or diaphragm movements between the acquisitions. A reduction of the rotation time to 0.35 s could probably decrease this isolated artefact.

Our results showed a SNR less pronounced in WV mode than helical, but in accordance with other studies $[3,16]$ it is negligible
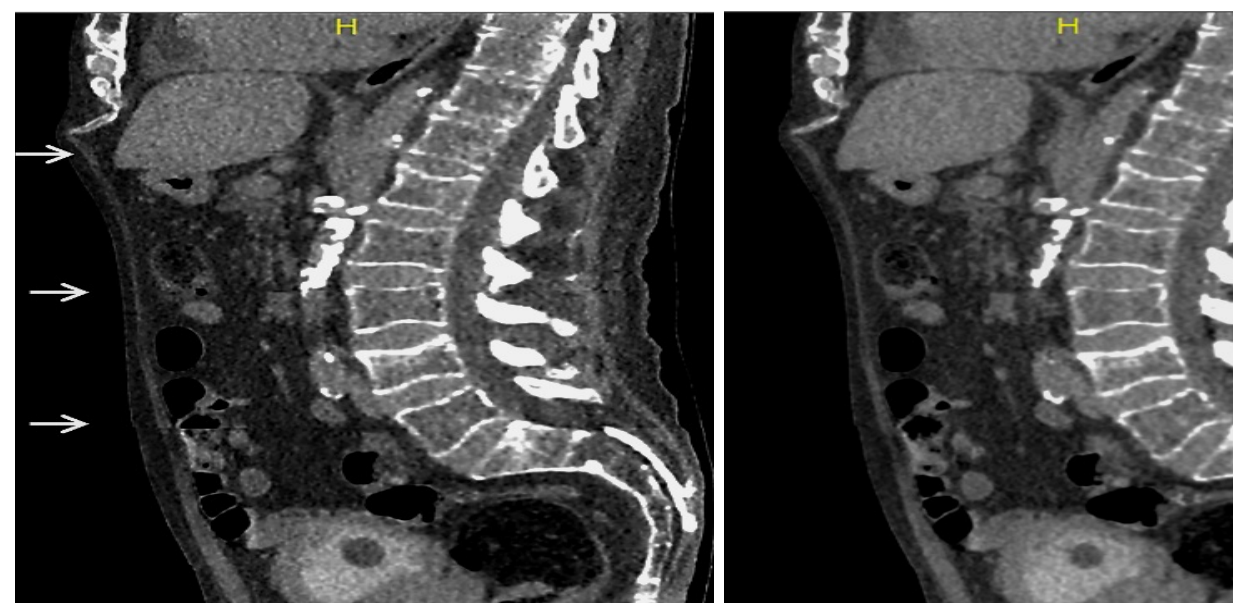

Figure 5a. Excretory WV mode -sagittal view -result of the automatic stitching (a1). The junctions between volumes are recognized at certain levels $(\rightarrow$ white arrow); after additional manual stitching (a2); the junctional zones have disappeared. 
Roy C (2017) Quantitative and qualitative evaluation of the Wide Volume (WV) versus Helical acquisition on a 320-detector row computed tomography for the exploration of the abdominopelvic region in adults
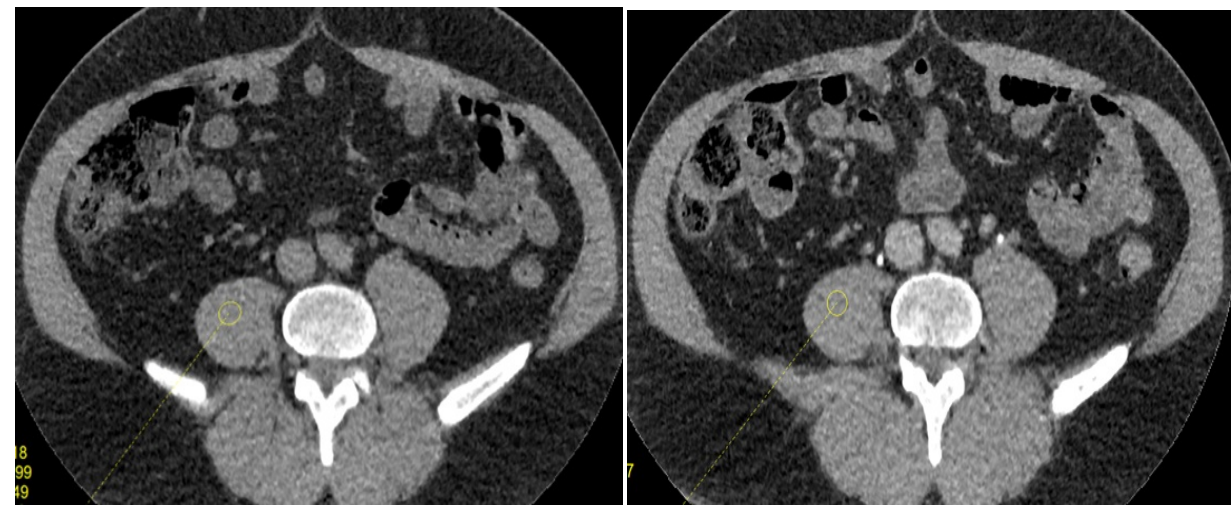

Figure 5b. Qualitative evaluation between helical acquisition unenhanced (b1) and excretory WV mode (b2). There is no visual difference for a clinical purpose between those two acquisition modes.

Figure 5. Qualitative evaluation

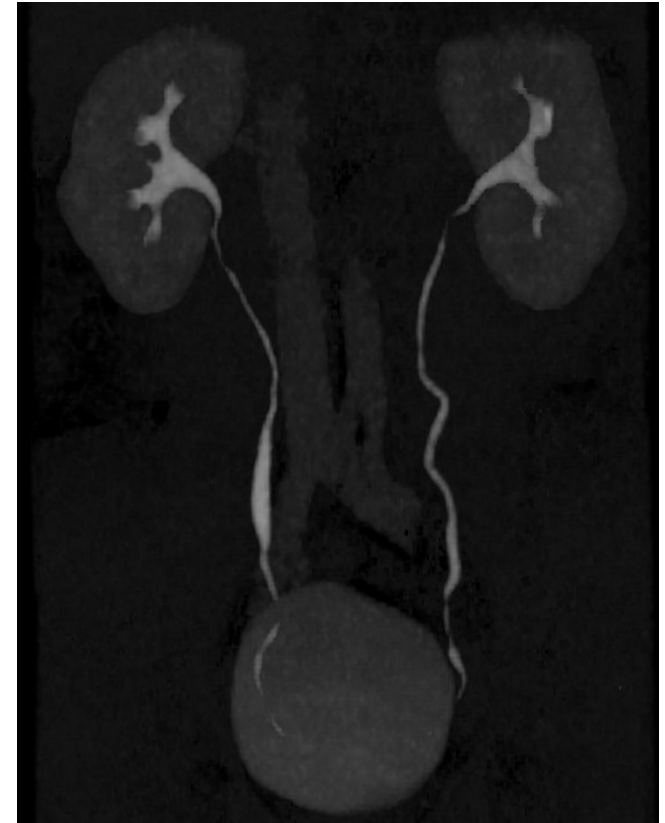

Figure 6a. Normal patient (follow up after ablation of a bladder excretory tumour)-antero posterior MIP projection. The ureters are entirely evaluated without disruption.

Figure 6. Clinical cases

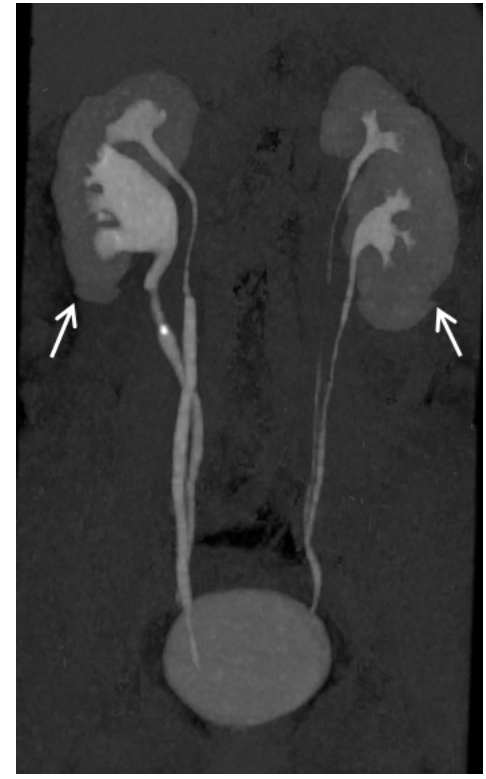

Figure 6b. Duplicated excretory system with a urinary stone in the upper part of the lower excretory system. The ureter above the stone is collapsed due to the pathological process Note that there is a discontinuity ( $\uparrow$ white arrow) of the lower pole of the kidneys, but it does not interfere for the right diagnosis. with respect to image quality. The diagnosis confidence was always preserved in our clinical study.

This study has some limitations.

-First, the SNR analysis using ROI positioned in the psoas area is not necessarily representative of the overall quality of the acquisition; however, this technique is commonly used in the literature. The score used to define the quality of volume fusion is subjective, but the ureter is a very easy anatomical structure to follow.

-Second, the dose profile curves were acquired with a radiationsensitive film and an optical scanner. This is a semi-quantitative technique. We did not have at our disposal a digital system that could have been more precise.

\section{Conclusion}

Using WV acquisition mode on a 320 detectors row CT scan to image the abdominopelvic cavity in adults with the collimation of 10 $\mathrm{cm}(200 \times 0.5 \mathrm{~mm})$ instead of $16 \mathrm{~cm}(320 \times 0.5 \mathrm{~mm})$ strongly reduces the radiation dose with an acceptable scanning time, while keeping an excellent image quality. It can be used for a diagnosis purpose.

The dose reduction is related to various factors: the lack of over ranging, a lower over beaming and better modulation in the $\mathrm{Z}$-axis when using several smaller volumes. It can be recommended in routine clinical practice.

\section{Disclosures of conflicts of interest}

No potential conflicts of interest, including financial, consultant, institutional or other relationships to disclose.

\section{References}

1. Khan AN, Shuaib W, Nikolic B, Khan MK, Kang J, et al. (2014) Absorbed Radiation Dose in Radiosensitive Organs Using 64- and 320-Row Multidetector Computed Tomography: A Comparative Study. Scientifica article ID 305942, 6 pages, doi:10.1155/2014/305942 
Roy C (2017) Quantitative and qualitative evaluation of the Wide Volume (WV) versus Helical acquisition on a 320-detector row computed tomography for the exploration of the abdominopelvic region in adults

2. Kroft LJ, Roelofs JJ, Geleijns J (2010) Scan time and patient dose for thoracic imaging in neonates and small children using axial volumetric 320-detector row CT compared to helical 64-, 32-, and 16-detector row CT acquisitions. Pediatr Radiol 40: 294-300. [Crossref]

3. Gervaise A, Louis M, Batch T, Loeuille D, Noel A, et al. (2010) Dose reduction at $\mathrm{CT}$ of the lumbar spine using a 320-detector row scanner : initial results (french). $J$ Radiol 91 : 779-785. [Crossref]

4. Yamashiro T, Miyara T, Takahashi M, Kikuyama A, Kamiya H, et al. (2012) Lung image quality with 320-row wide-volume CT scans: the effect of prospective ECGgating and comparisons with 64-row helical CT scans. Acad Radiol 19: 380-388. [Crossref]

5. Chen MY, Shanbhag SM, Arai AE (2013) Submillisievert median radiation dose for coronary angiography with a second-generation 320-detector row CT scanner in 107 consecutive patients. Radiology 267: 76-85. [Crossref]

6. Ryu YJ, Kim WS, Choi YH, Cheon JE, Lee SM, et al. (2015) Pediatric Chest CT: Wide-Volume and Helical Scan Modes in 320-MDCT. AJR Am J Roentgenol 205: 1315-1321. [Crossref]

7. Johnston JH, Podberesky DJ, Yoshizumi TT, Angel E, Toncheva G, et al. (2013) Comparison of radiation dose estimates, image noise, and scan duration in pediatric body imaging for volumetric and helical modes on 320-detector CT and helical mode on 64-detector CT. Pediatr Radiol 43:1117-1127. [Crossref]

8. de Denaro M, Bregant P (2011) Dosimetric evaluation of a 320 detector row CT scanner unit. Radiol Oncol 45: 64-67. [Crossref]
9. Khan A, Nasir K, Khosa F, Saghir A, Sarwar S, et al. (2011) Prospective gating with 320-MDCT angiography: effect of volume scan length on radiation dose. AJR Am J Roentgenol 196: 407-411. [Crossref]

10. Kalra MK, Maher MM, Toth TL, Schmidt B, Westerman BL, et al. (2004) Techniques and applications of automatic tube current modulation for CT. Radiology 233: 649-657. [Crossref]

11. Liao YL, Lai NK, Tyan YS, Chuang KS, Tsai HY (2013) Automatic tube curren modulation for volume scan in a 320-detector row CT scanner. Radiation Measurements 56: $328-332$.

12. Deak PD, Langner O, Lell M, Kalender WA (2009) Effects of adaptive section collimation on patient radiation dose in multisection spiral CT. Radiology 252: 140-147. [Crossref]

13. Perisinakis K, Papadakis AE, Damilakis J (2009) The Effect of X-Ray Beam Quality and Geometry on Radiation Utilization Efficiency in Multidetector CT Imaging. Med Phys 36: 1258-1266. [Crossref]

14. Liao YL, Chen YS, Lai NK, Chuang KS, Tsai HY (2014) Overbeaming and overlapping of volume-scan CT with tube current modulation in a 320-detector row CT scanner. Radiation Physics and Chemistry 104: 225-229.

15. Siebert E, Bohner G, Dewey M, Masuhr F, Hoffmann KT, et al. (2009) 320-slice CT neuroimaging: initial clinical experience and image quality evaluation. $\mathrm{Br} J$ Radiol 82 :561-570. [Crossref]

16. Bauknecht HC, Siebert E, Dannenberg A, Bohner G, Jach C, et al. (2010) Image quality and radiation exposure in 320-row temporal bone computed tomography. Dentomaxillofac Radiol 39: 199-206. [Crossref]

Copyright: (C2017 Roy C. This is an open-access article distributed under the terms of the Creative Commons Attribution License, which permits unrestricted use, distribution, and reproduction in any medium, provided the original author and source are credited. 